\title{
The Analysis of Stratified Teaching of Applied Mathematics in Higher Colleges \\ Xinai Zhang ${ }^{1}$ \\ ${ }^{1}$ Baoding Vocational College of Science and Technology, Baoding, Hebei, 071051 \\ hunter2011@foxmail.com
}

Keywords: Stratified Teaching, Applied Mathematics, Higher Colleges

\begin{abstract}
The diversity of the college students has increased the gap between their math foundations and capabilities, thus it's an inexorable trend to implement stratified teaching in the subject of "applied mathematics". The phrase "stratified teaching" means to separate students into groups according to their math degrees and interests, and give each group a suitable teaching plan. This article discussed the concept and necessity of "stratified teaching", and introduced the implementation measures concerning teaching and learning practices.
\end{abstract}

\section{Introduction}

In recent years, with the rapid development of China's colleges, the size is increasing and the education problems is also aappearing. Compared with students in vocational colleges and universities, cultural base is relatively poor, learning consciousness is weak, but they are highly personalized, hobbies wider difference between individuals is very obvious. In mathematics learning, learning level is uneven levels. Therefore, in the teaching of mathematics in vocational application, we must fully consider the student's mathematical foundation and learning levels, select appropriate teaching content and the use of appropriate teaching methods, teaching students at different levels in order to achieve vocational schools Applied Mathematics the purpose and effectiveness.

"Stratified teaching mode" from the "individualized" teaching the principles of Confucius proposed that the sub-classes early speed, high school is essentially different. Essence "hierarchical teaching model" is the premise of the class system, take different depending on the basis of student teaching, teaching staging achieves similar goals. This teaching method is to respect students' individuality, including effective measures for the common development of all students. American psychologist Bloom said: "Many students in the study failed to achieve excellent results, the main problem is not the lack of students' intellectual ability, but because did not receive proper teaching conditions and reasonable assistance caused by the". "If the provision of appropriate learning conditions, most of the students in learning ability, learning speed, motivation to learn, and many more will become very similar." [1]

\section{The Meaning of the Stratified Teaching}

The so-called sub-level teaching is that teachers respect the students based on the current level of knowledge, ability level and development potential, rational grouping of students. Teachers in the teaching process, for different levels of groups were treated differently, individualized, so that students get the greatest progress in learning. China's current level teaching includes teaching objects at different levels, teaching at different levels, hierarchical teaching objectives, teaching content at different levels in several ways.

Mathematics teaching at vocational colleges, mainly based on the students' level of mathematics, students are divided into different levels, to develop appropriate level of teaching objectives, students at different levels using different teaching methods for teaching them. This is the individualized teaching philosophy embodied in the teaching of higher vocational education. 


\section{The Necessity of Implementation of Stratified Teaching Mode in Higher Mathematics}

In recent years, college enrollment and continuous popularization of higher education, the overall quality of students in higher vocational colleges decreased significantly [2].

First, from the student's mathematical foundation and interest in learning, the student is the source of higher vocational colleges. Survey results show: Vocational school students' math scores 50 points or less accounted for $82.2 \%, 51-75$ minutes of students accounted for 17.7 percent, 75 points or more students did not (out of 150 points). Because of this result on the basis of students' learning of mathematics is not interested in differences ability to understand mathematical knowledge, acceptance and other aspects is an objective reality;

Second, from the current textbook perspective, the current Higher Mathematics Textbook Although continuous reform, but not enough thorough, systematic requirements on theoretical and also too strong, computing power is too high, exercises and review questions large, and students generally poor foundation;

Third, from a professional point of view, most of the students' professional light basis weight "," Learning math does not go "," learn also useless ", resulting in" teaching "and" learning "in trouble, increasing polarization, resulting in Some students weariness, the knowledge gap is growing.

Mathematical foundations of vocational college students and current teaching situation, cannot guarantee all students in same step. Therefore, this teaching mode is not well implement the "individualized, step by step" principle, but also greatly dampened the enthusiasm of the majority of students, but also seriously affect the improvement of the quality of vocational education. However, no matter how different students can divide and fertility and it is one of the channels is to take the implementation of the hierarchical teaching model for teaching.

\section{The Specific Implementation of Stratified Teaching Mode in Higher Mathematics}

Teaching objectives stratified. Professional numerous species of higher vocational colleges, the application of mathematical ability requirements are different emphases, different. Economics and management professional requirements stresses mathematics, auto repair, bridge professional to learn engineering mathematics, computer professionals to learn linear algebra. Therefore, vocational education classes are generally compiled based on professional series of classes, students cannot be considered on the basis of the different situations in mathematics, there are many practical problems and difficulties during the whole hierarchical teaching. Based on the foundations of mathematics case of students in vocational colleges questionnaire, taking into account the vast majority of students the mathematical basis of the status quo is not very good, the sub-curricular teaching hierarchical level should not be too much, generally can be divided into two levels. That set the minimum classroom teaching objectives and expand teaching objectives, according to the teaching of the knowledge structure and cognitive ability of students, the knowledge, ability and integrated way of thinking, rational development of students at all levels of teaching objectives and target levels throughout all aspects of teaching. The teaching objectives are divided into four levels: memorizing, understanding, simple application, simple integrated application. For different levels of students, teaching objectives and requirements are not the same: the minimum requirements for students to achieve teaching objectives before the three requirements and expand the teaching objectives require students to achieve all of the requirements. For example: In a lecture on "derivation formula and derivation rules" when a section is required to improve the level of students bearing in mind the formulas and rules, can directly apply the formula and rules seeking a simple function of the derivative, and require students to expand the level of understanding of derivation of the formula, proficiency in the use formulas and rules to solve more integrated function derivation problems [3].

Teaching subjects stratified. First, we must create a good environment, good teacher-student relationship can create a good learning environment to stimulate student interest in learning, so that students' mental development. The purpose is to allow different levels of teaching different levels of students to maximize their potential to gradually narrow the gap, achieve overall optimization. Next, 
after the freshmen, after understanding school achievement, classroom observation, testing, and take students on a voluntary basis, the students in accordance with 8: 2 ratio divided into two levels:

One is the increasing layer (minimum teaching target layer). The majority of students as teaching objects minimum teaching objectives, this part of the students' basic knowledge of mathematics, although in general, but to learn more conscious, there is a certain degree of ambition, achievement medium or lower. The other is the development layer (expansion goals layer). Set minority students with good basis as the teaching objectives, this part of the students consciously, more solid foundation, strong ability to accept.

Teaching stratified Learning in the classroom is a two-way communication, to mobilize the enthusiasm of bilateral activities is the key to complete the different levels of teaching. Classroom teaching process to fully take into account each student's learning level base and capacity, must be $80 \%$ increase in the proportion of students as the base layer, taking into account $20 \%$ of the development level of students, intended to mobilize them to participate in teaching activities ratio, not As Snub. All the basic knowledge and competency requirements clearly advertised, but also to expand the knowledge explicitly tell them what? They need to learn what mathematical abilities and qualities? Trying to take into account the different levels of students to ensure that students can learn at different levels have income. Always observe classroom teaching gradual, easy to difficult, from simple to complex, and gradually increase the law requires is not too high. As far as possible convergence seamless transition from old knowledge to new knowledge, natural, structured.

Evaluation methods stratified. Set the minimum teaching objectives is not only the focus of teaching should also be clear to the students and this is the focus of coursework and examination. After the completion of teaching a unit or module, students should be consolidated and improved through a lot of practice, therefore, according to different levels of students learning ability, arranged different level teaching homework is an indispensable link. For this reason, in order to improve the basic layer operations based (the exercises), while there are a few simple applications with the title (after-school exercise), expanding layer job to be completed on the basis of section review questions of title. So we can fully mobilize the enthusiasm of students, reduce the academic burden on all students, reducing the phenomenon of copying operations, can also make the students' attention and learning spare capacity on the application of mathematical techniques and mathematical culture to develop, improve student learning mathematics interest and confidence.

After completing the course content of each chapter, to arrange a clearance examination, for different levels of students learning level design synthesis papers, textbook exercises it mainly focuses on the basic concepts and basic skills test. Among them, $80 \%$ of basic questions, raise issues accounted for $20 \%$. By using a larger value on the basis of the papers reduce the difficulty of the questions, and enhance the confidence of students weak foundation, but with a certain proportion of expanding the subject, the students played a specialty, let generate a sense of accomplishment, inspire them to learn the high number of road on going.

\section{The Issues Should be Noted in the Implementation of Stratified Teaching Mode in Higher Mathematics}

"Stratified teaching mode" teaching objectives, teaching subjects, teaching process, evaluation methods, etc. hierarchy is important, but also pay attention to the following aspects: First, focus only on achievement levels, contempt ability cultivation; second level points too dead, increased polarization; Third, pay attention to only part of eugenics, ignoring all students; four students structured, single teacher teaching; teaching stratification and fifth test is not complete. These negative factors in the teaching practice, we should pay attention to overcome [4].

"Stratified teaching mode" from the traditional harmonization, standardization, mechanization, instead diversification, hierarchy and personalization, in order to achieve the establishment of a unified teaching requirements and individual advantages to develop new teaching requirements for the teaching objectives, so that different students get the maximum level of development is becoming 
a reality; teaching content because students have the appropriate level of change; also create a better environment for the student body of the play, the students have truly become masters of learning.

Stratified teaching mode has higher requirements for teachers and needs more teachers' workload. Teachers need to have a strong sense of responsibility, truth-seeking, innovative style of work. Properly used in Higher Mathematics Teaching in "Stratification Teaching", allows students a clearer purpose, consciousness stronger, more intense interest in learning, to reduce polarization, large area to improve mathematics teaching quality. Hierarchical teaching is a difficult operation of the new work, to be explored and improved in future practice. However, it should be noted that: In today's focus on "people-oriented", to promote lifelong learning, personalized learning, independent study of social trends, multi-faceted multi-level hierarchical teaching is undoubtedly correct. It is an important symbol of the traditional teaching to modern teaching change. Enable students to establish self awareness and career awarenessas as soon as possible and improve their self-awareness and social competence [5].

\section{Conclusion}

Stratified teaching mode of Applied Mathematics at the Higher Colleges is an education reform, it is still in the developmental exploratory stage and there are some problems and shortcomings inevitably, which need continuous exploration and summary in the teaching process. We are hoping to find a more scientific and more rational teaching mode for higher vocational colleges.

\section{References}

[1] Y.Tang, Exploration and practice of higher mathematics stratified teaching, J. Jiaxing University, 5 (2007) 59-61.

[2] N.Yang, The rational thinking of mathematics teaching at the university, J. Higher Education Forum, 4(2007) 129-132.

[3] A.J.Yao, Asynchronous layered teaching in mathematics teaching at the university, J. Chinese Vocational Education. 9(2009) 19-22.

[4] X.J.Chen. Exploration and practice of higher mathematics stratified teaching. 11(2006) 26-30.

[5] J.G.Sun, The strategies of classroom difference stratified teaching, J. China Education Daily, 5(2007) 46-47. 